\title{
Designing for multiple global user populations: increasing resource allocation efficiency for greater sustainability
}

\author{
Nadadur, G.and Parkinson, M.B. ${ }^{*}$ \\ Department of Mechanical and Nuclear Engineering, The Pennsylvania State University. \\ 213 Hammond Building, University Park, PA 16802, USA.
}

\begin{abstract}
This paper proposes a method to identify opportunities for increasing the efficiency of raw material allocation decisions for products that are simultaneously targeted at multiple user populations around the world. The values of 24 body measures at certain key percentiles were used to estimate the best-fitting anthropometric distributions for female and male adults in nine national populations, which were selected to represent the diverse target markets multinational companies must design for. These distributions were then used to synthesize body measure data for combined populations with a 1:1 female:male ratio. An anthropometric range metric (ARM) was proposed for assessing the variation of these body measures across the populations. At any percentile, ARM values were calculated as the percentage difference between the highest and lowest anthropometric values across the considered user populations. Based on their magnitudes, plots of ARM values computed between the $1^{\text {st }}$ and $99^{\text {th }}$ percentiles for each body measure were grouped into low, medium, and high categories. This classification of body measures was proposed as a means of selecting the most suitable strategies for designing raw material-efficient products. The findings in this study and the contributions of subsequent work along these lines are expected to help achieve greater efficiencies in resource allocation in global product development.
\end{abstract}

Keywords: anthropometry, design for sustainability, designing for multiple user populations, design for human variability

\section{Introduction}

This paper presents a simple technique for effectively comparing anthropometric data for multiple user populations of products that are marketed globally (e.g., automobiles, industrial workstations, commercial aircraft, apparel). The anthropometric range metric (ARM) is developed to this end. The metric makes for easy assessment of the variation of body measures across user populations, and is used to identify potential means of increasing raw material allocation efficiency in design decision-making.

Rapidly increasing levels of globalization are resulting in increasing numbers of product categories (e.g., vehicles, apparel, consumer electronics, industrial workstations) that are simultaneously targeted at multiple countries [30]. As a result, the target user populations of these products differ in terms of dis- tributions of anthropometry (body measures), among other characteristics. Body dimensions are a key determinant of an individual's "fit" in a design and the individual's reach, strength, and other general capabilities [16]. Relevant body measures influence users' preferred ways of physically interacting with products. When the user is able to interact with a product in ways that are comfortable and satisfying to the user and also safe for the user as well as other individuals in the usage area, the user is considered $a c$ commodated by the design of the product. Knowledge about relevant anthropometry is therefore crucial to achieving the desired accommodation level of the target user population of the product at hand.

In global product development, in order to achieve the desired accommodation level of each target population, designers are compelled to offer products in different sets of sizes and adjustability ranges in each

\footnotetext{
*Corresponding author. E-mail: parkinson@psu.edu. Telephone: +1 (814) 863-9079.
} 
region of interest (e.g., East Asia, Europe, the United States, etc.). Methodologies to increase commonalities across the different designs of globally marketed products could be helpful in increasing the efficiency of the development and manufacturing processes for certain products (e.g., industrial workstations, aircraft seating). This paper looks to identify opportunities for the development of such methodologies through a study of anthropometric information for multiple national populations.

Numerous design approaches use human models in accommodation analyses of designs. The human models are sized to represent the variation of body measures within the target population. Boundary manikin approaches involve the use of human models, which are sized to represent the lower and upper percentiles ( $5^{\text {th }}$ and $95^{\text {th }}$, for example) of the population [23]. A more robust alternative, which allows for better capturing of the anthropometric variability within the population, is to create a family of manikins [3]; the product is considered to achieve the required accommodation level if every one of these manikins fits into the design.

However, more accurate accommodation analyses may be conducted through the application of user behavior simulations yielded by quantitative virtual fitting methods. The statistical models used in these simulations are formulated based on data obtained through usage experiments involving the interaction of sample groups of users with prototypes of the product. User anthropometry plays an important role in these models, and is used to predict usage behaviors $[6,10]$. The experiment-based models allow for the consideration of variability in user behavior due to differences in preferred ways of physically interacting with products. Despite the differences in procedures, complexity, and accuracy across these methodologies, all of them are based on the anthropometry of the user population.

Anthropometric databases can be good sources of information about the required body measures for the target population. However, comprehensive anthropometric databases, which are compiled through surveys of the different body measures for representative samples of an entire population, are few in number. Furthermore, due to the investments of time, labor, money, and other resources required, these databases are difficult to develop. ANSUR [12] and NHANES [20] are examples of existing databases that are representative of certain user populations (the U.S. military in 1988 and U.S. civilians, respectively). In contrast, the CAESAR [4] population, which is composed of randomly selected North American and European participants, is not representative of an actual user population, but may be appropriately reweighted and transformed to a more representative database.

There are many examples of representative and non-representative databases which are the result of surveys conducted around the world: Brazil's SERPRO survey [17], China's Human Dimensions of Chinese Adults [28], India's survey of agricultural workers [11], Japan's Human Engineering for Quality of Life [15], Germany's MikroZensus [26], and England's Health Survey [27]. Software such as PeopleSize [21] can provide designers with information about anthropometry at key percentiles for a number of countries and user populations. Each target population is unique in terms of descriptors that may be demographic (e.g., age variation, gender split) or others (income and education level, nutrition level and healthcare, occupation and physical exertions, etc.). Since population anthropometry is influenced by these descriptors, every user population is likely to be characterized by unique distributions of body measures. This leads to varying design decisions across multiple target populations of products. In a global design context, greater awareness of anthropometric similarities and differences across populations can allow designers to identify ways in which to apply existing ergonomic design principles and techniques to improve resource efficiency.

Different strategies may be applied to make products robust to the anthropometric variability within and across populations. These strategies include the practices of sizing and adjustability allocation, product family development, and reconfigurable design. A number of research efforts (e.g., [2, 22]) have examined the application of adjustability and sizing analyses in enabling a design to efficiently accommodate the desired percentage of the target population. Studies have demonstrated the incorporation of adjustability in the design of products such as industrial workstations [8, 14].

Designing reconfigurable products has been the focus of some recent research efforts. The development of reconfigurable designs is based on metrics that assess the type and amount of flexibility required in the product $[7,9]$. Using a suitable methodology, the product can even be designed to be modifiable, and thereby adaptable to changing user populations $[13,25]$. 
I Available anthropometric data Summary stats at certain percentiles

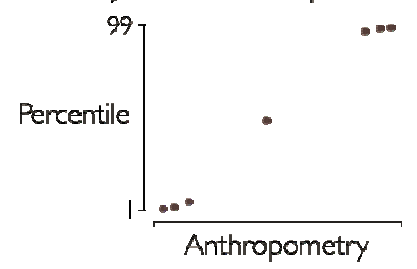

II Optimally fitted distributions Normal or log-normal distributions

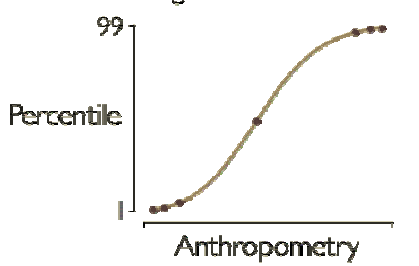

III Anthropometry range metric Comparing anthropometry across populations

ARM Eqn. (1)

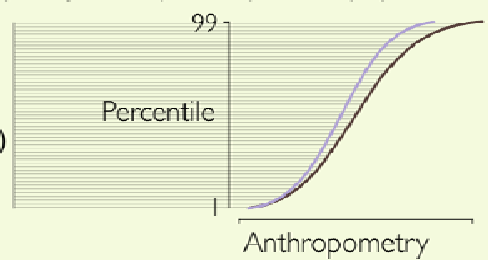

Figure 1: The three-step methodology implemented in this work. Summary statistical information about the anthropometry is gathered for the different populations in Step I. In Step II, optimal normal and log-normal distributions are fitted to the summary stats of length- and breadth/circumference- related body measures, respectively. Step III involves using the anthropometry range metric (ARM) to compare the variation of each body measure across the nine populations.

Research in the area of designing product families and platforms makes for increased variety at reduced costs [24]. Customer preferences, market competition, and other relevant factors can be taken into account in formulating appropriate product platforming strategies [19]. Tools such as the design structure matrix, value analysis, and the commonality versus diversity index can be used to examine existing product families and identify ways to improve them [1]. Welldesigned product variants enable the designer to efficiently satisfy a range of requirements in the marketplace.

This work compares 24 body measures for nine national civilian target populations---Germany, Italy, Japan, Kenya, South Korea, the Netherlands, Thailand, the United States, and China---of a product that is marketed globally (e.g., automobiles, industrial workstations, commercial aircraft, apparel). These populations were chosen to represent the diversity in markets and user populations a multinational company might choose to target. Easily available anthropometric information for these populations usually consists of only the values of certain body measures at a few percentiles. Section 2 describes the methodology implemented to first estimate the required anthropometric distributions for the chosen populations, and to then compare these distributions using the anthropometry range metric. Section 3 presents

As shown in Table 1, the anthropometry chosen for this study included 16 measures of length and 8 measures of breadth/circumference. It should be noted that the information for some of the nine populations did not include these statistics for all 24 body measures. Furthermore, the age ranges of the surveyed populations varied across the nine countries of interest.

In Step II of the methodology, female and male summary statistics were first considered separately. the results of this analysis, and discusses certain observations from the computed ARM values. Section 4 summarizes the paper and presents the conclusions.

\section{Methodology}

The basic three-step methodology is illustrated in Figure 1. As mentioned earlier, the only data available for the chosen populations were the values of the body measures at certain key percentiles (Step I). In order to more thoroughly compare the anthropometry, normal or log-normal distributions were fitted to these summary statistics (Step II). The newly proposed ARM measure was then used to thoroughly compare these distributions between the $1^{\text {st }}$ and $99^{\text {th }}$ percentiles for the nine user populations (Step III). This allowed for a better understanding of the variability of anthropometry across the populations.

Summary statistics for the Chinese population were obtained from the State Bureau of Technical Supervision survey [28], and the statistics for the other 8 populations were from a report compiled by the International Organization for Standardization [18]. These statistics consist of separate sets of values of body measures at certain key percentiles for women and men in the population.

Due to their correlation to stature, measures of length are known to be approximately normally distributed [5]. Similarly, measures of breadth/circumference, which are correlated to weight, tend to be log-normal in nature $[5,28]$. Using this knowledge and the aforementioned percentile information, the mean and standard deviation were calculated for the best-fit normal or log-normal distribution for each body measure in every population. The distribution was fitted to minimize the sum of the absolute value of the errors between the actual and estimated anthro- 
pometry at each of the key percentiles. This yielded the optimal mean and standard deviation for each distribution. The body measures estimated from the resulting distribution were found to be the close approximations of the actual information.
The anthropometry range metric (ARM) was used in Step III of the methodology to assess the variation of body dimensions across the populations.

At each percentile $p$ between 1 and 99, ARM was calculated as the scaled difference

Table 1

A matrix that lists the availabilities of the 24 body measures for the 9 national user populations. Also shown is the age range of the surveyed population that is the basis for the summary stats.

\begin{tabular}{|c|c|c|c|c|c|c|c|c|c|}
\hline & \multicolumn{9}{|c|}{ Populations } \\
\hline & $\begin{array}{c}\text { Germa- } \\
\text { ny }\end{array}$ & Italy & Japan & Kenya & $\begin{array}{l}\text { South } \\
\text { Korea }\end{array}$ & $\begin{array}{c}\text { The } \\
\text { Nether- } \\
\text { lands }\end{array}$ & Thailand & U.S. & China \\
\hline Age range (years) & $18-65$ & $18-65$ & $20-65$ & $18-73$ & $18-60$ & $18-65$ & $30-39$ & $18-65$ & $18-55$ \\
\hline \multicolumn{10}{|l|}{ Anthropometry } \\
\hline \multicolumn{10}{|l|}{ Lgth-related } \\
\hline Butt-Knee Lgth & $\sqrt{ }$ & $\mathbf{x}$ & $\sqrt{ }$ & $\sqrt{ }$ & $\sqrt{ }$ & $\sqrt{ }$ & $\sqrt{ }$ & $\sqrt{ }$ & $\mathbf{x}$ \\
\hline Butt-Pop Lgth & $\sqrt{ }$ & $\sqrt{ }$ & $\sqrt{ }$ & $\sqrt{ }$ & $\sqrt{ }$ & $\sqrt{ }$ & $\sqrt{ }$ & $\mathbf{x}$ & $\sqrt{ }$ \\
\hline Elbw-Wrist Lgth & $\mathbf{x}$ & $\sqrt{ }$ & $\sqrt{ }$ & $\sqrt{ }$ & $\sqrt{ }$ & $\mathbf{x}$ & $\sqrt{ }$ & $\mathbf{x}$ & $\mathbf{x}$ \\
\hline Eye Ht, Sit & $\sqrt{ }$ & $\mathbf{x}$ & $\sqrt{ }$ & $\sqrt{ }$ & $\sqrt{ }$ & $\sqrt{ }$ & $\sqrt{ }$ & $\sqrt{ }$ & $\sqrt{ }$ \\
\hline Eye Ht, Stand & $\sqrt{ }$ & $\mathbf{x}$ & $\sqrt{ }$ & $\sqrt{ }$ & $\sqrt{ }$ & $\sqrt{ }$ & $\sqrt{ }$ & $\mathbf{x}$ & $\sqrt{ }$ \\
\hline Foot Lgth & $\sqrt{ }$ & $\sqrt{ }$ & $\sqrt{ }$ & $\sqrt{ }$ & $\sqrt{ }$ & $\sqrt{ }$ & $\sqrt{ }$ & $\sqrt{ }$ & $\sqrt{ }$ \\
\hline Forward Reach & $\sqrt{ }$ & $\mathbf{x}$ & $\sqrt{ }$ & $\sqrt{ }$ & $\sqrt{ }$ & $\sqrt{ }$ & $\sqrt{ }$ & $\mathbf{x}$ & $\mathbf{x}$ \\
\hline Hand Lgth & $\sqrt{ }$ & $\sqrt{ }$ & $\sqrt{ }$ & $\sqrt{ }$ & $\sqrt{ }$ & $\sqrt{ }$ & $\sqrt{ }$ & $\sqrt{ }$ & $\sqrt{ }$ \\
\hline Head Lgth & $\sqrt{ }$ & $\sqrt{ }$ & $\sqrt{ }$ & $\sqrt{ }$ & $\sqrt{ }$ & $\sqrt{ }$ & $\mathbf{x}$ & $\sqrt{ }$ & $\sqrt{ }$ \\
\hline Knee Ht & $\sqrt{ }$ & $\sqrt{ }$ & $\sqrt{ }$ & $\sqrt{ }$ & $\sqrt{ }$ & $\sqrt{ }$ & $\sqrt{ }$ & $\sqrt{ }$ & $\mathbf{x}$ \\
\hline Popliteal Ht & $\sqrt{ }$ & $\sqrt{ }$ & $\sqrt{ }$ & $\sqrt{ }$ & $\sqrt{ }$ & $\sqrt{ }$ & $\sqrt{ }$ & $\mathbf{x}$ & $\sqrt{ }$ \\
\hline Shldr-Elbw Lgth & $\sqrt{ }$ & $\sqrt{ }$ & $\sqrt{ }$ & $\sqrt{ }$ & $\sqrt{ }$ & $\sqrt{ }$ & $\mathbf{x}$ & $\mathbf{x}$ & $\mathbf{x}$ \\
\hline Shldr Ht, Sit & $\sqrt{ }$ & $\sqrt{ }$ & $\sqrt{ }$ & $\sqrt{ }$ & $\sqrt{ }$ & $\sqrt{ }$ & $\mathbf{x}$ & $\sqrt{ }$ & $\sqrt{ }$ \\
\hline Shldr Ht, Stand & $\sqrt{ }$ & $\sqrt{ }$ & $\sqrt{ }$ & $\sqrt{ }$ & $\sqrt{ }$ & $\sqrt{ }$ & $\sqrt{ }$ & $\sqrt{ }$ & $\sqrt{ }$ \\
\hline Sit Ht & $\sqrt{ }$ & $\sqrt{ }$ & $\sqrt{ }$ & $\sqrt{ }$ & $\sqrt{ }$ & $\sqrt{ }$ & $\sqrt{ }$ & $\sqrt{ }$ & $\sqrt{ }$ \\
\hline Stature & $\sqrt{ }$ & $\sqrt{ }$ & $\sqrt{ }$ & $\sqrt{ }$ & $\sqrt{ }$ & $\sqrt{ }$ & $\sqrt{ }$ & $\sqrt{ }$ & $\sqrt{ }$ \\
\hline \multicolumn{10}{|l|}{ Brth-/Circ-related } \\
\hline Biacromial Brth & $\sqrt{ }$ & $\sqrt{ }$ & $\sqrt{ }$ & $\sqrt{ }$ & $\sqrt{ }$ & $\sqrt{ }$ & $\sqrt{ }$ & $\sqrt{ }$ & $\sqrt{ }$ \\
\hline Bideloid Brth & $\sqrt{ }$ & $\sqrt{ }$ & $\sqrt{ }$ & $\sqrt{ }$ & $\sqrt{ }$ & $\sqrt{ }$ & $\sqrt{ }$ & $\sqrt{ }$ & $\sqrt{ }$ \\
\hline Chest Circ & $\sqrt{ }$ & $\sqrt{ }$ & $\sqrt{ }$ & $\sqrt{ }$ & $\sqrt{ }$ & $\sqrt{ }$ & $\sqrt{ }$ & $\sqrt{ }$ & $\sqrt{ }$ \\
\hline Foot Brth & $\sqrt{ }$ & $\sqrt{ }$ & $\sqrt{ }$ & $\sqrt{ }$ & $\sqrt{ }$ & $\sqrt{ }$ & $\sqrt{ }$ & $\sqrt{ }$ & $\mathbf{x}$ \\
\hline Head Brth & $\sqrt{ }$ & $\sqrt{ }$ & $\sqrt{ }$ & $\sqrt{ }$ & $\sqrt{ }$ & $\sqrt{ }$ & $\sqrt{ }$ & $\sqrt{ }$ & $\sqrt{ }$ \\
\hline Hip Brth, Sit & $\sqrt{ }$ & $\sqrt{ }$ & $\sqrt{ }$ & $\sqrt{ }$ & $\sqrt{ }$ & $\sqrt{ }$ & $\sqrt{ }$ & $\sqrt{ }$ & $\sqrt{ }$ \\
\hline Hip Brth, Stand & $\sqrt{ }$ & $\mathbf{x}$ & $\sqrt{ }$ & $\sqrt{ }$ & $\sqrt{ }$ & $\mathbf{x}$ & $\sqrt{ }$ & $\mathbf{x}$ & $\sqrt{ }$ \\
\hline Thigh Circ & $\sqrt{ }$ & $\mathbf{x}$ & $\sqrt{ }$ & $\sqrt{ }$ & $\sqrt{ }$ & $\mathbf{x}$ & $\sqrt{ }$ & $\mathbf{x}$ & $\mathbf{x}$ \\
\hline
\end{tabular}




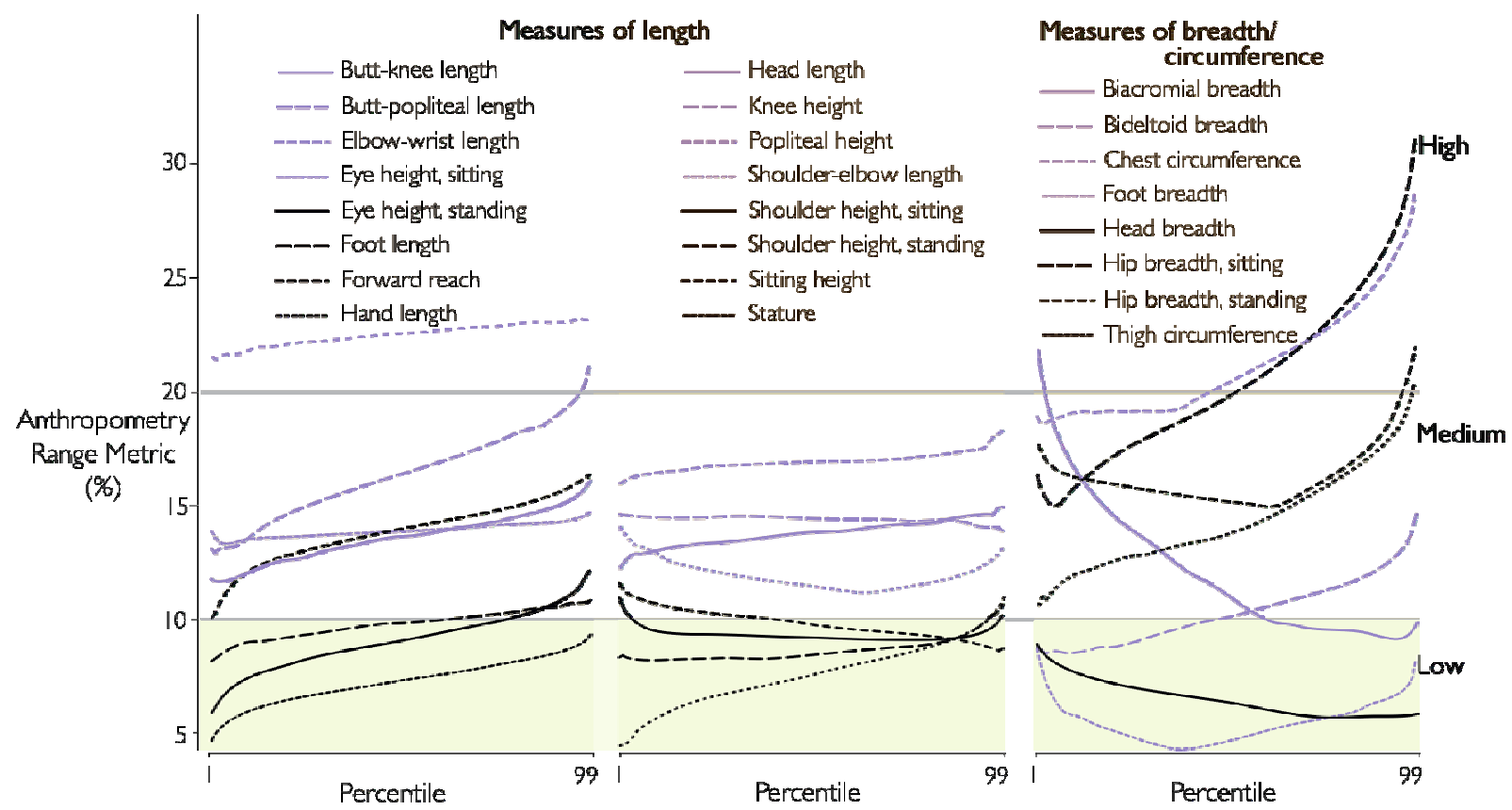

Figure 2: Plots of the anthropometry range metric (ARM) calculated for the 24 body measures and for the female users in the nine populations.

between the maximum and minimum values of the anthropometry ( $\mathbf{A}_{p, \max }$ and $\mathbf{A}_{p, \text { min }}$, respectively) across the populations:

$$
\operatorname{ARM}(p)=100 .\left(\mathbf{A}_{p, \text { max }}-\mathbf{A}_{p, \text { min }}\right) / \mathbf{A}_{p, \text { mean }}
$$

where $\mathbf{A}_{p \text {,mean }}$ is the mean of the values of that body measure at the $p^{\text {th }}$ percentile for the nine populations. The resulting ARM values were scaled percentages, and allowed for the application of some common analysis guidelines across all 24 body dimensions. Figures 2 and 3 show the ARM plots between the $1^{\text {st }}$ and $99^{\text {th }}$ percentiles for the female and male populations.

Next, for each national population, the female and male anthropometric distributions from Step II were randomly sampled to create a combined population of users with a 1:1 female:male ratio. This was done to facilitate more realistic anthropometric assessments, since designs are very rarely intended for only users of a particular sex. Step III of the process was then once again applied, with Equation (1) used to calculate ARM values for the resulting data. Figure 4 contains plots of these ARM values.

\section{Results and discussion}

The ARM plots in Figures 2, 3, and 4 allow for easy visual assessments of the anthropometry in the nine national populations. The figures are divided into high, medium, and low regions of ARM values. ARM plots in the low region signify smaller variations (less than 10\%) in body measure values across the populations. Plots in the medium region indicate greater variations (between 10\% and 20\%), and those in the high region exhibit variations above $20 \%$.

Figures 2 and 3 allow for comparisons of the anthropometric variations in the female and male populations. Certain differences can be observed for the body measures. ARM values for sitting shoulder height are in the medium region for the male population, and are mostly low for the female populations. Hand length values are low for females, but largely medium for males. The plots of bideltoid breadth and sitting height are in the medium region for males, and partly in the medium region and partly in the low region for females. Thigh circumference values are in the medium range for females, but are largely low for males. Biacromial breadth plots are partly high and 


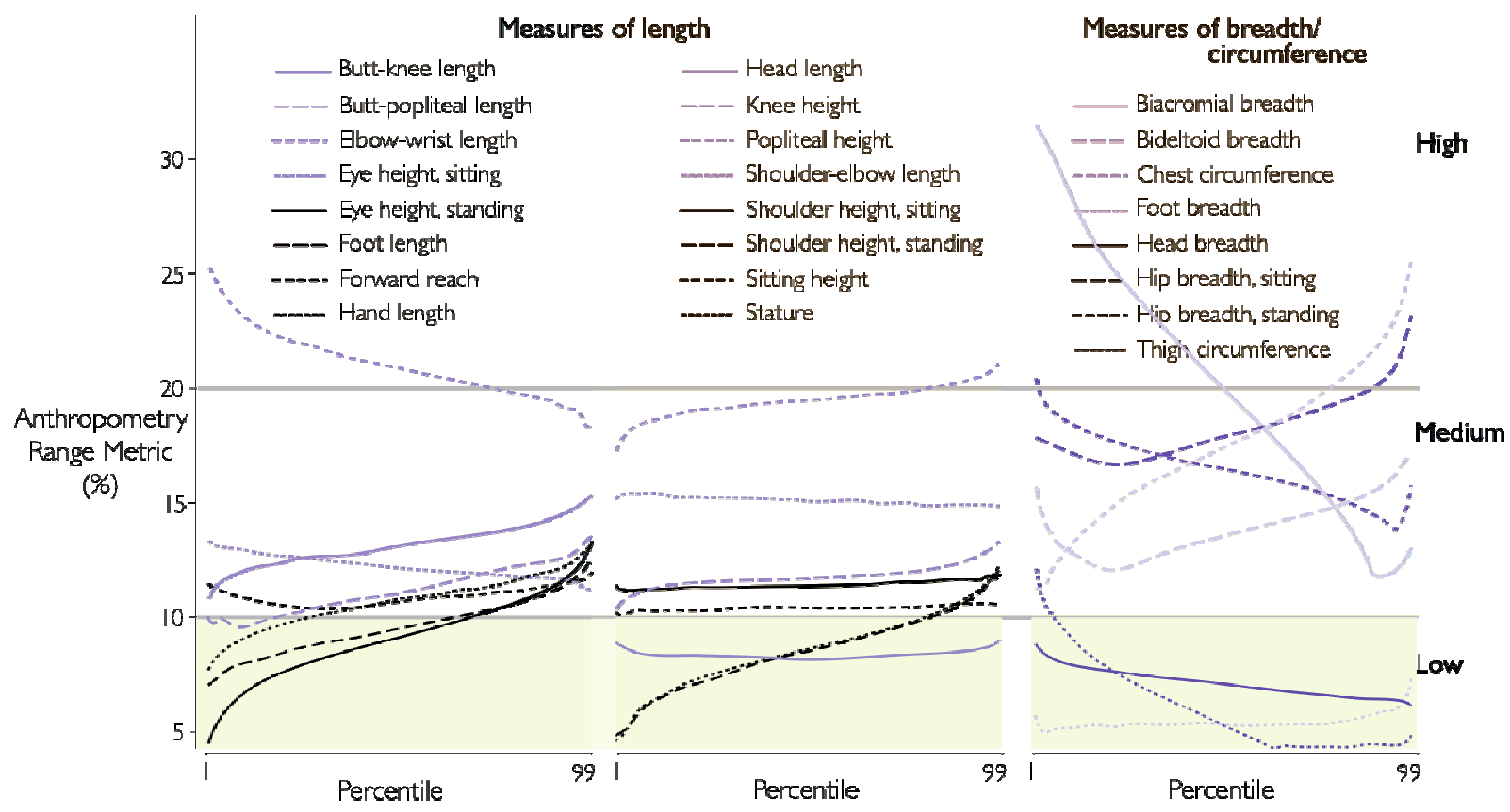

Figure 3: Plots of the anthropometry range metric (ARM) calculated for the 24 body measures and for the male users in the nine populations.

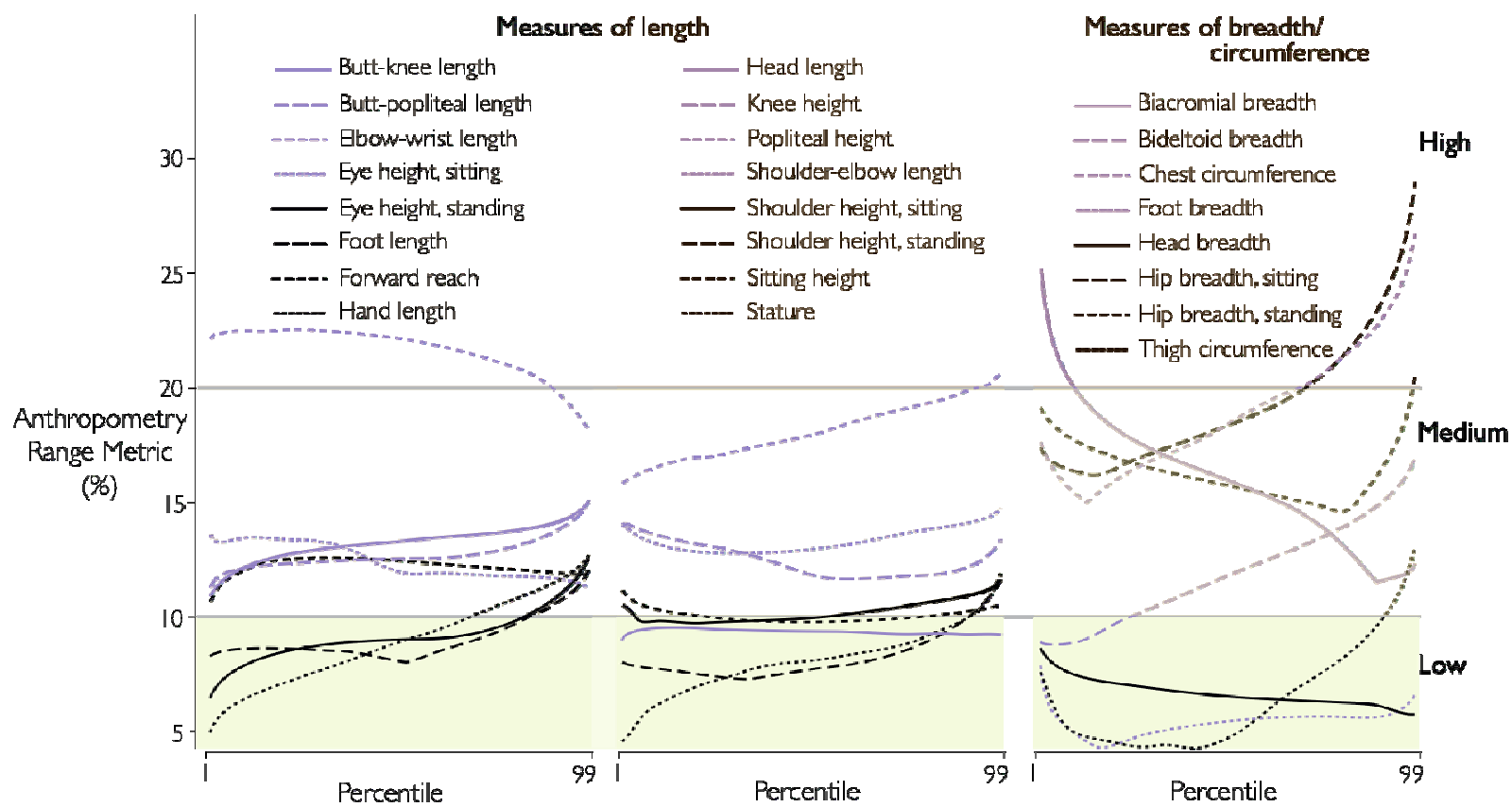

Figure 4: Plots of the anthropometry range metric (ARM) calculated for the 24 body measures and for the nine national populations with a $1: 1$ female:male ratio. 
partly medium for males, and span the high, medium, and low regions for females.

The ARM plots in Figure 4, which are based on the nine combined populations, enable assessments of anthropometric variations across the entire populations. The ARM plots for head length, head breadth, and foot breadth are completely within the low region. The plots for standing eye height, foot length, standing shoulder height, stature, and thigh circumference are almost entirely in the low region; they extend into the medium region at the upper tails of their distributions. These plots indicate relatively low variation in the values of the corresponding body measures across the nine populations.

The ARM values for buttock-popliteal length, sitting eye height, standing eye height, forward reach, knee height, and shoulder-elbow length are in the medium region. The plots of biacromial breadth, bideltoid breadth, standing hip breadth, and popliteal height extend into the high and low regions at the tails of their distributions. Elbow-wrist length is mostly in the high region.

The ARM plots of chest circumference, hand length, and sitting hip breadth are more evenly divided into two different regions. Also, the plots of certain body measures are more erratic in nature. Sitting shoulder height and sitting height are in the medium region at the lower tails of their distributions, then move into the low region, and finally extend back up into the medium region.

The proposed anthropometry range metric is found to be a simple and effective way to quantify and understand the anthropometric variability encountered across multiple user populations. This measure will be used extensively in future work into resourceefficient global product developmental practices.

\subsection{Future work}

The observations made using the newly proposed ARM measure indicate the potential for the application of different strategies in the development of products that are simultaneously marketed in multiple user populations around the globe. For instance, optimal sizing and adjustability allocation could be the means to designing components of the product that interact with the body measures that are in the low region of Figure 4. If the relevant body measures are in the medium region, then reconfigurability could allow for more efficient use of resources. Product platforming could be the most suitable strategy for body measures in the high region. Future research will be aimed at exploring these design strategy implications of anthropometric variability.

There are certain limitations to these analyses, which will also be the foci of future work. First, the division of Figures 2, 3, and 4 into the three ARM regions---below 10\%, 10-20\%, and above $20 \%$---was arbitrary. Ongoing research on this project is aimed at proposing a more objective method for doing so. Second, the assumption of 1:1 female:male ratio may not be valid for the user population of every category of products or for users in every country. Third, and most importantly, the procedure used in synthesizing anthropometry for the nine national populations does not allow for more detailed, multivariate design analyses involving more than one body measure. Given the dearth of detailed databases of body measures for more populations and taking into account the availability of summary statistics for numerous populations, this was the simplest and most efficient way to estimate the required data. Ongoing efforts are aimed at developing a method to use summary statistics to accurately synthesize anthropometric data for entire virtual populations that are statistically equivalent to the actual populations.

\section{Conclusion}

This research utilized summary statistical information to estimate optimal distributions for 24 body measures in nine different national populations: Germany, Italy, Japan, Kenya, South Korea, the Netherlands, Thailand, the United States, and China. The anthropometry range metric (ARM) was applied on the resulting distributions, and was used to quantify the anthropometric variability between the $1^{\text {st }}$ and $99^{\text {th }}$ percentiles for the 24 body measures across the 9 populations. The ARM measure has been demonstrated as being a useful tool in this context, and will be utilized in the development of a method for suitable strategy selection based on the anthropometric variability encountered in different design scenarios. Future research along these lines is expected to yield decision-making tools for the more efficient development of products intended for multiple global markets.

\section{Acknowledgments}

This research was partially funded by the National Science Foundation under Award No. 0846373. Any 
opinions, findings, and conclusions or recommendations expressed in this material are those of the author(s) and do not necessarily reflect the views of the National Science Foundation.

\section{References}

[1] F. Alizon, S.B. Shooter, T.W. Simpson, Improving an existing product family based on commonality/diversity, modularity, and cost, Design Studies, 28 (2007), 387-409.

[2] A. Bhattacharya, J.D. McGlothlin, Occupational ergonomics: Theory and applications, CRC Press, $1^{\text {st }}$ Edition, 1996.

[3] A.C. Bittner, A-CADRE: Advanced family of manikins for workstation design, Proceedings of the Human Factors and Ergonomics Society, (2000), 774-777.

[4] S. Blackwell, T. Brill, M. Boehmer, S. Fleming, S. Kelly, D. Hoeferlin, K. Robinette, CAESAR survey measurement and landmark descriptions, Air Force Research Laboratory, Wright-Patterson Air Force Base, Ohio, USA, 2008.

[5] J. Brainard, D.E. Burmaster, Bivariate distributions for height and weight of men and women in the United States, Risk Analysis, 12 (1992), 267-275.

[6] K. Case, M. Porter, D. Gyl, R. Marshall, R. Oliver, Virtual fitting trials in 'design for all', Journal of Materials Processing Technology, 117 (2001), 255-261.

[7] P. Cormier, D. Van Horn, K. Lewis, Investigating the use of reconfigurability to reduce product family cost and mitigate performance losses, ASME Design Engineering Technical Conferences (DETC2009-87439), ASME International, San Diego, CA, USA, 2009.

[8] B. Das, A.K. Sengupta, Industrial workstation design: A systematic ergonomics approach, Applied Ergonomics, 27 (1996), $157-163$.

[9] S. Ferguson, A. Siddiqi, K. Lewis, O.L. de Weck, Flexible and reconfigurable systems: Nomenclature and review, ASME Design Engineering Technical Conferences (DETC200735745), ASME International, Las Vegas, NV, USA, 2007.

[10]C.J. Garneau, M.B. Parkinson, A comparison of methodologies for designing for human variability, Journal of Engineering Design, 22 (2011), 505-521.

[11]L.P. Gite, J. Majumdar, C.R. Mehta, A. Khadatkar, Anthropometric and strength data of Indian agricultural workers for farm equipment design, All India Coordinated Research Project on Ergonomics and Safety in Agriculture, Central Institute of Agricultural Engineering, Bhopal, India, 2009.

[12]C.C. Gordon, T. Churchill. C.E. Clauser, B. Bradtmiller, J.T. McConville, I. Tebbetts, R.A. Walker, 1988 Anthropometric survey of U.S. army personnel: Methods and summary statistics, Final report, U.S. Army Natick Research, Development, and Engineering Center, NATICK/TR-89/027, 1989.

[13]J. Haldaman, M.B. Parkinson, Framework for the incorporation of reconfigurability in design for human variability, Journal of Engineering Design (in review), 2010.
[14]C.D. Hochandel, Computer workstation adjustment: A novel process and large sample study, Applied Ergonomics, 26 (1995), 315-326.

[15] Human Engineering for Quality Life, Japanese body size data 1992-1994, Research Institute of Human Engineering Quality Life, Osaka, Japan, 1997.

[16]Human Factors and Ergonomics Society 300 Committee, Guidelines for using anthropometric data in product design, Human Factors and Ergonomics Society, Santa Monica, California, USA, 2004.

[17] Instituto Nacional de Tecnologia, ERGOKIT, Divisao de Desenho Industrial, Instituto Nacional de Tecnologia, Brazil, 1998.

[18] International Organization for Standardization, ISO/TR 72502:2010, 2010, url: $\mathrm{http}: / / \mathrm{www}$.iso.org,iso/catalogue_detail?csnumber=41249.

[19]D. Kumar, W. Chen, T.W. Simpson, A market-driven approach to the design of platform-based product families, AIAA/ISSMO Multidisciplinary Analysis and Optimization Conference, 2006.

[20]National Center for Health Statistics, National health and nutrition examination survey (NHANES), U.S. Centers for Disease Control and Prevention, 2008, url: http://www.cdc.gov/nchs/nhanes.htm.

[21] Open Ergonomics Ltd., PeopleSize software, 2008, url: http://www.openerg.com/psz/

[22] S. Pheasant, C.M. Haslegrave, Bodyspace: Anthropometry, ergonomics, and the design of work, Taylor \& Francis, $3^{\text {rd }}$ Edition, 2006.

[23] Siemens PLM Software, Tecnomatix Jack, 2009.

[24] T.W. Simpson, Z. Siddique, R.J. Jiao, Product platform and product family design, Springer Science+Business Media, Inc. New York, NY, USA, 2005.

[25] V. Singh, S.M. Skiles, J.E. Krager, K.L. Wood, D. Jensen, R. Sierakowski, Innovations in design through transformation: A fundamental study of transformation principles, Journal of Mechanical Design, 131 (2009).

[26] Statisches Bundesamt Deutschland, Mikrozensus, 2004. url: http://www.destatis.de/jetspeed/portal/cms/Sites/destatis/Inter net/DE/Presse/abisz/Mikrozensus.templateId=renderPriint.ps $\mathrm{ml}$.

[27] The NHS Information Center, Health survey for England, Department of Health, England, 2009, url: http://ww.dh.gov.uk/en/Publicationsandstatistics/PublishedSur vey/HealthSurveyForEngland/index.htm.

[28] The State Bureau of Technical Supervision, Human dimensions of Chinese adults (GB 10000-88), 1989.

[29] J.L. Veerman, J.J. Barendregt, E.F. van Beeck, J.C. Seidell, J.P. Mackenbach, Stemming the obesity epidemic: A tantalizing prospect, Obesity, 15 (2007), 2365-2370.

[30] M.F. Wiersema, H.P. Bowen, Corporate diversification: The impact of foreign competition, industry globalization, and product diversification, Strategic Management Journal, 29 (2008), 115-132. 